\title{
SCALA: Towards a physical calibration of CALSPEC standard stars based on a NIST-traceable reference for SNIFS
}

\author{
D. Küsters ${ }^{1}{ }^{2}$ S. Lombardo ${ }^{3},{ }^{2}$, M. Kowalski ${ }^{1}{ }^{2}$, G. Aldering ${ }^{4}$, \\ K. Boone ${ }^{4}$, Y. Copin ${ }^{5}$, J. Nordin ${ }^{2}$ and D. Rubin 6 \\ ${ }^{1}$ Deutsches Elektronen-Synchrotron, D-15735 Zeuthen, Germany \\ ${ }^{2}$ Institut fur Physik, Humboldt-Universitat zu Berlin, Newtonstr. 15, 12489 Berlin \\ email: kuesters@physik.hu-berlin.de \\ ${ }^{3}$ Aix Marseille Univ, CNRS, CNES, LAM, Marseille, France \\ ${ }^{4}$ Physics Division, Lawrence Berkeley National Laboratory, Berkeley, CA \\ ${ }^{5}$ Universit de Lyon, Institut de Physique Nuclaire de Lyon \\ ${ }^{6}$ Space Telescope Science Institute, Baltimore, MD
}

SCALA is a physical calibration device for the SuperNova Integral Field Spectrograph (SNIFS), mounted to the University Hawaii $2.2 \mathrm{~m}$ telescope on Mauna Kea. For type Ia supernova (SN Ia) cosmology programs, an improved fundamental calibration directly translates into improved cosmological constraints. The aim of SCALA is to perform a fundamental calibration of the CALSPEC (Bohlin 2014) standard stars, which are currently calibrated relative to white dwarf model atmospheres.

SCALA transfers the calibration of a flux calibrated photodiode to SNIFS (Lombardo 2017). As the photodiode is sensitive over a broad wavelength range, a monochromator lamp setup is used to perform the transfer wavelength by wavelength. This calibration of SNIFS relative to a laboratory flux-calibrated broad-band detector can be compared with the usual SNIFS calibration relative to the CALSPEC standard stars, and consequently ties the laboratory detector standard with the models for the white dwarf atmospheres.

We report on the characterization of several systematics, which we correct our measurements for, most prominently internal reflections and out-of-band emission from our monochromator (Küsters 2018). We compare our results to the existing CALSPEC system (model atmospheres) and to a compilation of Vega flux calibrations using laboratory-calibrated light sources (Hayes 1985). Our measurements agree with the CALSPEC and Hayes 1985 results within $\pm 2 \%$ in a wavelength range from $4500 \AA$ to $9000 \AA$.

We plan to improve the baffling of our light source and detector to suppress reflections internal to SCALA. We further plan to use a double monochromator to suppress the out-of-band emission. Both improvements should lead to sub-percent uncertainties in the calibration of the standard stars and an increased wavelength range, from $3500 \AA$ to $9900 \AA$.

\section{References}

Bohlin, R. C. 2014, The Astronomical Journal, 147.6, 127

Lombardo, S. et al 2017, Astronomy and Astrophysics, 607, A113

Küsters, D. 2018, Humboldt Universität zu Berlin, Phd Thesis

Hayes, D. S. 1985, Symposium-International Astronomical Union, 111, 225-252 